\title{
Does urbanization promote rising house prices?
}

\author{
Yunyun Luo \\ School of Economics and Management, Beijing Jiaotong University, Beijing, China \\ 17120551@bjtu.edu.cn
}

Keywords: Urbanization level, House prices, Panel data, Regional differences.

\begin{abstract}
With the acceleration of urbanization process and fluctuations in real estate prices, housing problem has become an urgent problem that hinders the improvement of people's living standards. Based on the panel data of 31 provinces (excluding Hong Kong, Macao and Taiwan) in China from 2001 to 2016, this paper established a spatial econometric model to analyze the impact of urbanization on housing prices. It found that there are regional differences in the impact of urbanization on housing prices. there are obvious positive effects in the eastern and central regions, negative effects in western regions, and no significant effects in northeast China. In addition, the current house price is also affected by factors such as the previous house price, per capita GDP, and household consumption level. This study is helpful to understand the relationship between urbanization level and housing prices, and maintain the stable estate prices in various regions.
\end{abstract}

\section{Introduction}

Since the reform and opening up, China's economy has developed rapidly, and people's living standards have improved remarkably. The main manifestation is that a large number of people gather in cities, and the scale of cities is expanding. China's urbanization process has experienced three stages. From taking the development of big cities as the center to coordinated development of large, medium and small cities and small towns, and then to new urbanization development, we pay more and more attention to human integration. With the improvement of urbanization level, real estate prices have shown a volatility, and scholars have done a lot of research on this topic.

On the one hand, scholars have discussed the reasons for the rise in housing prices. Restricting land supply will reduce the supply of new housing and rental housing, which will eventually lead to both rent and housing prices rising [1]. In the United States, land-use controls limit the amount of land available for development, thereby increasing land prices, which ultimately leads to a decline in the supply of new housing and high house prices [2]. The monopoly of land supply of China's local government pushed up land prices [3]. It in turn pushed up house prices [4]. The increase in land sales has pushed up land prices. cities that rely more on land finance and the pressure on the price of residential land are also increasing [5].

On the other hand, scholars believe that the level of urbanization is closely related to housing prices. M.R. Ren and B. Liu used provincial panel data to find that the level of urbanization has a positive impact on housing prices, and the increase in urbanization will lead to a rapid rise in housing prices [6]. X. Deng and H.M. Kong used the data of housing prices and urbanization rate in China from 1998 to 2010, and used GMM estimation method to find that the urbanization level pushed the housing prices up [7]. L.G. Luo and Y.R. Pan believed that the level of urbanization in the country is uneven, and the difference in urbanization level has brought different degrees of influence on regional housing prices [8].

It can be found that the level of urbanization does have an impact on housing prices, providing a basis for the selection and research of the text. China's geographical differences are extremely great. Therefore, this paper divides China into four major economic regions, eastern, central, western and northeast, and adds control variables such as house prices, per capita GDP, and household consumption levels in the previous year to establish panel regression. The model, a more comprehensive and scientific way to reveal the impact of urbanization on housing prices, is significant for promoting the coordinated development of China's real estate industry and urbanization. 


\section{Variable selection and empirical model setting}

\subsection{Variable selection}

The selected variable data must meet the principles of typicality, conciseness, and operability, and can be selected from existing standard databases.

(1) Interpreted variables

The house price (real estate price) refers to the market value of the building along with the land occupied by the building in a certain period of time, and is the most important adjustment mechanism for real estate economic operation and resource allocation. This paper selects the average selling price of the house as the indicator of the house price level. In order to enhance the stability of the data, the growth rate is expressed by GLFA.

(2) Explanatory variables

This study is about the impact of urbanization on housing prices, so the explanatory variable is an indicator of urbanization level, which is used to measure the degree of urbanization development. It is generally expressed by the proportion of urban population in a certain area to the total population. The proportion of the population to the total population (URB) is used as an explanatory variable.

(3) Control variables

The current house price is also affected by factors such as the previous house price, so this paper selects the previous period house price growth rate (GLFA (-1)), per capita GDP growth rate (GGDP), and household consumption level index (GLNC) as control variables.

\subsection{Model setting}

Regression analysis is a statistical analysis method that determines the quantitative relationship between two or more variables. In order to verify the impact of urbanization on housing prices, this paper establishes the following regression model:

$$
\mathrm{GLFA}=\alpha+\beta_{1} \mathrm{URB}+\beta_{2} \mathrm{GGDP}+\beta_{3} \mathrm{GLNC}+\beta_{4} \mathrm{GLFA}(-1)+\varepsilon .
$$

In formula (1), GLFA indicates the growth rate of house price. URB indicates the level of urbanization. GGDP indicates the growth rate of GDP per capita. GLNC indicates the index of household consumption. GLFA (-1) indicates the growth rate of house price in the previous period. $\alpha$, $\beta_{1}, \beta_{2}, \beta_{3}$ and $\beta_{4}$ are parameters to be estimated, and $\varepsilon$ represents an error term.

\section{Empirical results and analysis}

This paper selects the relevant data of the eastern, central, western and northeastern regions of China from 2001 to 2016, and uses EVIEWS software for analysis. The data mainly comes from the following two channels. The first is the public database. The second is the China Statistical Yearbook (2017).

(1) Unit root test

Table 1. Unit root test result.

\begin{tabular}{clllc}
\hline Variable & LLC & ADF-Fisher & PP-Fisher & Conclusion \\
\hline GLFA & $-13.3275^{* * *}$ & $174.221^{* * *}$ & $325.681^{* * *}$ & Smooth \\
\hline GGDP & $-10.4029^{* * *}$ & $127.702^{* * *}$ & $382.353^{* * *}$ & Smooth \\
\hline GLNC & $-10.8077^{* * *}$ & $135.970^{* * *}$ & $353.765^{* * *}$ & Smooth \\
\hline URB & $-7.27584^{* * *}$ & $82.3273^{* * *}$ & $140.411^{* * *}$ & Smooth \\
\hline
\end{tabular}

Note: $(1) *, * *, * * *$ respectively indicate a hypothesis test at a confidence level of $10 \%, 5 \%$, and $1 \%$; (2) Take the eastern region as an example. The results in the rest of the region are similar, so they are omitted.

As can be seen from Table 1, the house price growth rate (GLFA), per capita GDP growth rate (GGDP), household consumption level index (GLNC), and urbanization rate (URB) are all tested by the unit root. The variables of the model are first-order, so, subsequent time series analysis can be performed between them. 
(2) Analysis from the vector regression model

The VAR model is a non-structural econometric model that is mainly used to predict the dynamic relationship between time series, and thus can explain the impact of various economic shock phenomena on economic variables. In the model, each equation can be estimated by the method OLS, and the assumption that the random interference term is not related to the sequence is omitted. Any sequence related phenomenon can be solved by adding the lag term of the interpreted variable. It was found that the growth rate of house prices (GLFA) was affected by the inertia of the previous period, and it had a positive effect on itself. However, the impact of per capita GDP growth rate (GGDP), household consumption level index (GLNC) and urbanization rate (URB) have no significant lag on housing prices. The AR unit root is performed below, and the result is shown in Fig. 1. It can be seen that all unit roots are located outside the unit circle, indicating that the VAR model satisfies the stability condition, that is, there is a long-term stable equilibrium relationship between the variables.

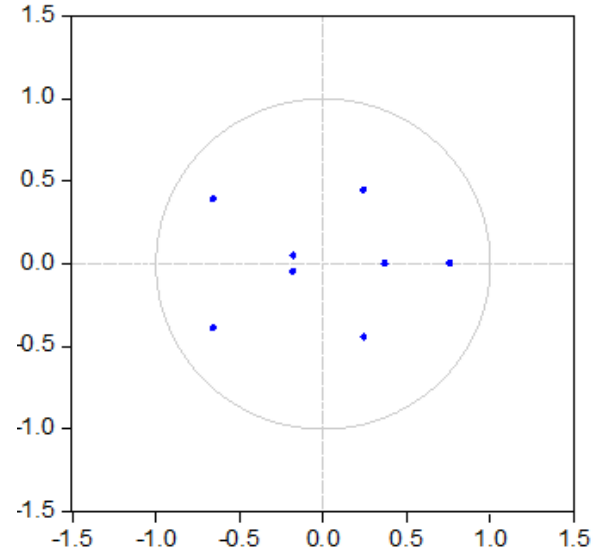

Fig. 1. Inverse Roots of AR Characteristic Polynomial

(3) Establish a regression model

The above part verifies the long-term stable equilibrium relationship between urbanization level and house price, and then establishes a regression model. The results are shown in Table 2.

Table 2. Regression results.

\begin{tabular}{ccccc}
\hline Areas & Eastern coastal area & $\begin{array}{c}\text { Central inland } \\
\text { area }\end{array}$ & Western areas & North-east area \\
\hline GGDP & $\begin{array}{c}1.641482^{* * *} \\
(8.093838)\end{array}$ & $\begin{array}{c}0.681651^{* * *} \\
(8.881427)\end{array}$ & $\begin{array}{c}0.256511^{* * *} \\
(3.188286)\end{array}$ & $\begin{array}{c}0.102474 * * * \\
(3.723359)\end{array}$ \\
\hline GLNC & $-1.461051^{* * *}$ & $-0.420178^{* * *}$ & $0.671872^{* * *}$ & -0.059767 \\
$(-2.869260)$ & $(-2.686493)$ & $(5.841437)$ & $-0.737576)$ \\
\hline URB & $1.432624^{* * *}$ & $0.582798^{* * *}$ & $-0.454062^{* * *}$ & -0.045956 \\
& $(2.632085)$ & $(3.923589)$ & $(-6.248438)$ & $(-0.952172)$ \\
\hline GLFA(-1) & $0.143820^{* *}$ & $-0.407605^{* * *}$ & $0.325985^{* * *}$ & $0.127181^{*}$ \\
& $(2.332449)$ & $(-7.001580)$ & $(7.230288)$ & $(1.833152)$ \\
\hline c & 0.313873 & 0.227249 & $-0.524280^{* * *}$ & $0.151721^{* *}$ \\
& $(0.335277)$ & $(1.146358)$ & $(-4.576363)$ & $(2.098984)$ \\
\hline Number of observations & 224 & 224 & 224 & 224 \\
\hline R ${ }^{2}$ & 0.330322 & 0.392569 & 0.629235 & 0.154694 \\
\hline Number of provinces & 10 & 6 & 12 & 3 \\
\hline
\end{tabular}

Note: *,**,** respectively indicate a hypothesis test at a confidence level of $10 \%, 5 \%$, and $1 \%$.

It can be seen from the whole empirical results that the level of urbanization is an important factor affecting the change of house prices, and there are regional differences in the degree of influence. The level of urbanization in the eastern region has a significant positive impact on housing prices. The reason may be that a large number of foreign laborers have moved in the east, and the demand for housing is strong, resulting in a situation in which supply is less than demand and a sharp rise in housing prices. In contrast, although the central region has also shown a significant positive effect, but the degree of improvement is not large, indicating that the development of all aspects of 
urbanization is relatively balanced and coordinated. The urbanization rate in the western region has a negative impact on housing prices. The reason may be that the western region is relatively backward, and the urbanization process is in its infancy. When the level of urbanization is raised, it can produce a certain agglomeration effect, causing the decline in housing prices. The impact of urbanization rate on housing prices in Northeast China is not significant. The reason may be that the level of urbanization is still at a relatively low level. Without the cooperation of industrial urbanization and land urbanization, the only population will not have too much impact on the economy. Then it will not have a significant effect on housing prices. In addition, the current house price is also affected by factors such as the previous house price, per capita GDP, and household consumption level.

\section{Conclusion and countermeasures}

This paper empirically analyzes the impact of urbanization level on housing prices by using panel data from different regions. According to the empirical results, the following conclusions can be drawn. First, the increase in per capita GDP growth rate and household consumption level can promote housing prices. Second, housing prices are vulnerable to the inertia of the previous period. It has a positive effect on itself. Third, the urbanization level of different regions has different impacts on housing prices. Control the urbanization process in the eastern coastal areas and accelerate the urbanization process in the western region can ensure the reasonable and stable operation of housing prices. At present, the development of China's regions is not balanced. The urbanization process is too fast or too slow leading to the fact that real estate prices cannot be coordinated with the people's living standards. Based on this, this paper proposes the following countermeasures.

(1) Grasp the urbanization process and implement a sustainable strategy. While correctly understanding the relationship between urbanization and housing prices, pay attention to the coordination of housing prices with per capita GDP and household consumption, and ensure that potential urban populations in first-tier cities can afford housing. Avoid the rich create a housing bubble. Focus on the construction of poor and remote areas, accelerate the process of urbanization, and let more people live in the house.

(2) Reduce the difference in urbanization development in the region. Due to the low urbanization rate in the central and western regions, it means that there is a large room for improvement in these two regions. At the same time, along with the rise of labor costs in the eastern region, a large number of industries will be transferred from the eastern coast to the central and western regions in the future. The central and western regions should seize this opportunity to accelerate the adjustment and upgrading of industrial structure and promote the urbanization rate in the central and western regions, narrowing the difference in the level of urbanization between regions. The government should moderately control the scale of development of large cities, especially the growth of permanent residents in cities. Develop small and medium-sized cities and towns, accelerate the pace of new rural construction, narrow the gap between urban and rural areas, and improve the living conditions of farmers to reduce the population from rural to urban areas.

(3) Increase the effective supply of land and develop satellite towns and cities. On the basis of the existing city scale, improving the effectiveness of land supply and further developing the construction of satellite towns and urban circles will not only broaden the supply channels for urban housing, but also ease the tight supply of land in central cities and curb housing prices. We can construct and improve the housing provident fund system and the affordable housing system and implement different housing supply policies for different income families. Give full play to the role of affordable housing, low-profit housing and low-rent housing.

(4) Use policy to influence public expectations. Based on the empirical findings of this paper, public expectations about housing prices may also trigger a sharp rise in housing prices. From this perspective, the measures to control the current rapid rise in housing prices should also include the use of policy adjustments to influence public expectations. 


\section{References}

[1] M. Lu, H.J. Ou and B.K. Chen, Rationality or bubble: an empirical study of urbanization, immigration and housing prices. World Economy, vol.37, pp. 30-54, 2014.

[2] E. L. Glaeser and B.A. Ward, The causes and consequences of land use regulation: Evidence from Greater Boston, Journal of Urban Economics, vol.65, pp. 265-278, 2009.

[3] J.M. Cai and S.Y. Cheng, Two-way Monopoly of Land Price and Distortion of Land Resource Allocation. Economic History, vol.11, pp. 75-80, 2010.

[4] X.Q. Ping and M.Y. Chen, Financing, land price and real estate price trend. World Economy, vol.7, pp. 3-10, 80, 2004.

[5] S.Q. Zheng and Z. Shi, Land and Housing Market under "Land Finance": Analysis of Local Government Behavior. Guangdong Social Sciences, vol.2, pp. 5-10, 2011.

[6] M.R. Ren and B. Liu, The Relationship between House Price and Urbanization-An Empirical Analysis Based on Provincial Panel Data. Southern Economy, vol.2, pp. 41-49, 2009.

[7] X. Deng and H.M. Kong, Research on the Relationship between Urbanization and House Price Based on Dynamic Panel Model. Statistics and Decision, vol.12, pp. 105-107, 2013.

[8] L.W. Luo and Y.R. Pan, An Empirical Analysis of the Influence of Regional Urbanization Difference on Real Estate Price. Statistics \& Decision, vol.5, pp. 131-134, 2015. 